\title{
Cytotoxicities of a 4-META/MMA-TBBO Resin against Human Pulp Fibroblasts
}

\author{
Seiichiro FUJISAWA ${ }^{1}$ and Toshiko A.TSUMI ${ }^{2}$ \\ ${ }^{1}$ Departments of Oral Diagnosis \\ ${ }^{2}$ Departments of Oral Physiology, Meikai University School of Dentistry, 1-1 Keyakidai, Sakado, Saitama, 3500283, Japan \\ Corresponding author, E-mail:fujisawa@dent.meikai.ac.jp
}

Received December 5, 2003/Accepted March 19, 2004

This study was designed to investigate the cytotoxicity of MMA (methyl methacrylate) and 4-META (4methacryloyloxyethoxycarbonylphthalic anhydride)/MMA with or without TBBO (tri- $n$-butylborane partially oxide), an initiator and TBBO alone against human pulp fibroblasts (HPF).

Cell viabilities were measured by MTT assay. The cytotoxicity of 4-META/MMA-TBBO was comparable with that of MMA-TBBO. TBBO showed higher cytotoxicity than 4-META/MMA. The cytotoxicity induction of a 4-META/MMATBBO resin may be preferably associated with TBBO.

Key words: Cytotoxicity, 4-META/MMA-TBBO, Human pulp fibroblasts

\section{INTRODUCTION}

A 4-META/MMA-TBBO resin which is composed of 4-META, MMA and TBBO is widely used in dentistry as a bonding agents ${ }^{1-3)}$. There have been many previous studies of cytotoxicity ${ }^{4-7)}$ and pulp responses ${ }^{8,9)}$. Since high concentrations of 4-META are not obtained in water due to its hydrophobicity, some studies have shown an adverse effect of 4META on pulp and cytotoxicity when this compound was solubilized at high concentrations in biological systems by methyl methacrylate or dimethyl sulfoxide $^{4-8)}$. We previously investigated the hemolytic activity of 4-META and 4-MET, a hydrolyzed product of 4-META, which suggested that the hemolytic activity of 4-MET was markedly smaller than that of $4-\mathrm{META}^{10)}$. In a 4-META/MMA-TBBO resin, 4-META and TBBO are contained by $5 \%$ and $9.2 \%$ $\mathrm{w} / \mathrm{w}$ in MMA, respectively. Therefore, the quantity of TBBO is about two-fold greater than 4-META. The molecular weight of TBBO, a partially oxidized $\mathrm{TBB}$, is unknown, therefore, it is difficult to compare the cytotoxicity of TBBO with 4-META or MMA. To clarify the cytotoxicity of a 4-META/MMATBBO resin toward pulp, in the present study, we examined the cytotoxic activity of 4-META/MMA or MMA with TBBO and TBBO alone against HPF cells.

\section{MATERIALS AND METHODS}

\section{Materials}

Monomer (4-META/MMA, Lot. No. VG3) and catalyst (TBBO, Lot. No. VE 42) (Sun Medical Co. Ltd. Moriyama Japan) were used. MMA (Tokyo Kasei Kogyo Co.) was further purified. The following chemicals and reagents were obtained from the indicated companies: fetal bovine serum (FBS) (JRH Biosciences, Lenexa, KS); Cell Titer 96 AQueous One solution of MTS[3-(4,5-dimethylthiazol-2-yl)-2,5diphenyltetrazolium bromide] (Promega Co. Madison, WI).

\section{Cell culture}

HPF cells were obtained from a 4-year-old female undergoing extraction of a supernumerary tooth. Informed consent was obtained from her parents. Ethical clearance for the study was obtained similarily to that previously reported ${ }^{11)}$. The tissue was cut into 1 to $2 \mathrm{~mm}^{3}$ pieces, washed twice in phosphate-buffered saline (PBS) supplemented with $100 \mathrm{U} / \mathrm{mL}$ penicillin and $100 \mu \mathrm{g} / \mathrm{mL}$ streptomycin, and placed into tissue culture dishes. The explants were incubated in culture medium consisting of $\alpha$ MEM, 30\% FBS, $100 \mathrm{U} / \mathrm{mL}$ penicillin, and $100 \mu \mathrm{g} / \mathrm{mL}$ streptomycin, at $37^{\circ} \mathrm{C}$ in a humidified atmosphere of $5 \% \mathrm{CO}_{2}$ in air. When outgrowth was observed in the cultures, the medium was replaced twice until the cells reached confluence. The cells were then detached from the monolayer by brief treatment with $0.05 \%$ trypsin/0.004 M EDTA and recultured in $\alpha$ MEM containing $30 \%$ FBS until confluent monolayers were again obtained. HPF cells were maintained with $\alpha$-MEM containing 10\% FBS. Cells between the fifth and seventh passages were used in the experiments described below.

\section{Cytotoxicity}

HPF cells were seeded into 96 -well plates at a density of $5 \times 10^{3}$ cells/well in $0.1 \mathrm{ml}$ of MEM with $10 \%$ FBS and cultured at $37^{\circ} \mathrm{C}$ for 2 days. Before the addition of test materials, the cells were washed twice with serum free medium. A stock solution of indicated concentrations of 4-META/MMA with TBBO, MMA 
with TBBO or TBBO was prepared in dimethyl sulfoxide (DMSO). Immediately after preparations, it was diluted with DMSO and added to the wells; and the cells were then incubated at $37^{\circ} \mathrm{C}$ for 24 hours. DMSO (1\%)-treated cells served as the control. The number of viable cells was determined by the MTS colorimetric assay ${ }^{12)}$. The value of the control (the absorbance at $492 \mathrm{~nm}$ ) is represented as a cell survival value of $100 \%$.

\section{Statistical analysis}

Student's t test was used to asses the statistical significance of differences.

\section{RESULTS}

Cytotoxicity of MMA/4-META and MMA with or without TBBO and TBBO alone

Results are shown in Fig. 1. The 0.25\% 4META/MMA was significantly more cytotoxic than its $0.125 \%$ counterpart. The $0.125 \%$ 4-META/MMA was comparable with the control. There was no significant difference in the cytotoxicity between MMA/4-META and MMA with TBBO. In contrast, the cytotoxicity of TBBO was significantly greater than that of 4-META/MMA or MMA with TBBO at each concentration level. That of $0.5 \%$ TBBO corresponded to approximately $50 \%$ viability.

\section{DISCUSSION}

For approximately 30 years, 4-META/MMA-TBBO resin has been investigated ${ }^{1-3)}$, but its characterization and biocompatibility are not fully understood.

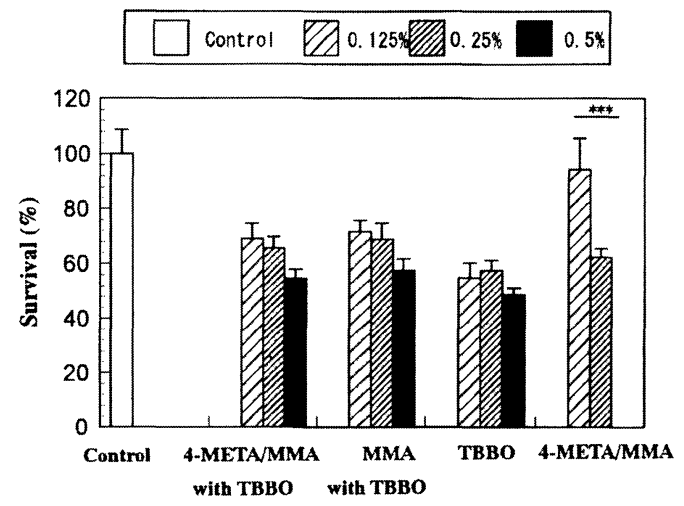

Fig. 1 The cytotoxicity of 4-META/MMA with or without TBBO and MMA with TBBO at different concentrations against human pulp fibroblasts (HPF). Bars indicate the mean $\pm \mathrm{SD} \quad(n=8)$. Results were analysed by Student's t-test. A significant difference of 4-META/MMA between $0.125 \%$ and $0.25 \%$ $(\mathrm{p}<0.001)$ and that between TBBO and 4META/MMA with TBBO $(\mathrm{p}<0.05)$ or MMA with TBBO $(p<0.05)$ at the indicated concentrations was found. Methods are described in the text.
To clarify the stability of 4-META in aqueous media our previously study suggested that 4-META was rapidly converted to 4 -MET, a hydrolyzed product of 4-META, in deuterium oxide using NMR spectroscopy ${ }^{10)}$. These findings agreed with those of spectroscopic experiments ${ }^{13)}$ using laser-Raman spectra of 4META in MMA solution. These findings suggested that 4-META with a carboxylic acid anhydride moiety is highly sensitive to moisture and is immediately converted to 4-MET with two carboxylic groups under clinical conditions even when carboxylic acid anhydride is retained until use.

We previously reported the strong hemolytic activity of 4-META and its large thermotropic alterations of L- $\alpha$-dipalmitoylphosphatidylcholine liposomes as a model for biomembranes ${ }^{10}$. However, the present study demonstrated that the cytotoxicity of 4META/MMA with TBBO was comparable with that of MMA with TBBO, suggesting that 4-META may not affect the cytotoxicity induction. In contrast, TBBO showed greater cytotoxicity than 4META/MMA and hence, the cytotoxic activity of 4META/MMA-TBBO may be responsible for TBBO. The cytotoxicity reduction of 4-META in the medium may be due to the fact that 4-META is rapidly converted to 4 -MET in water ${ }^{10}$ ) and from that 4-META preferably interacts with proteins ${ }^{14,15)}$ and alkaline earth salts ${ }^{17)}$.

The nerve regeneration and proliferative activity in amputated pulp tissue after the application of a 4-META/MMA-TBBO resin were previously reported $^{9)}$. This indicated that the healing activity of 4-META resin application was the same as that of calcium hydroxide, possibly due to boric acid derived from the decomposition of TBBO. From this it was clear that TBBO might be associated with much more pulp toxicity than 4-META.

The toxicity ${ }^{18)}$, cytotoxicity ${ }^{19)}$ and hemolytic activity $^{20)}$ of methacrylates are correlated with their hydrophobicity. In previous study ${ }^{21)}$, the values of hydrophobicity (log $\mathrm{P}_{\mathrm{HPLC}}$ ) for MMA, TBBO and 4-META were $0.79,0.005$, and 2.13 (and 1.23), respectively, using an HPLC. These values are related to those of octanol-water partition coefficient (log $\mathrm{P}$ ). MMA with 0.76 is dissolved by $1-1.3 \% \mathrm{w} / \mathrm{v}$ in water at room temperature. Despite that MMA is a volatile substance, this compound is relatively watersoluble, however, $50 \%$ cytotoxicity of MMA against HPF cells was about $30 \mathrm{mM}$ in this study, suggesting less cytotoxicity against HPF cells. Less cytotoxicity for MMA against human gingival fibroblasts (HGF) was previously reported ${ }^{21)}$. In contrast, 4-META shows two values of $\log \mathrm{P}_{\mathrm{HPLC}}$, suggesting that 4-META is 2.13, whereas 4-MET is 1.23 . As above mentioned, 4-META is probably converted to 4-MET in water, resulting from the study of HPLC. Thus, in the present study, the cytotoxicity for 4-META/MMA or MMA with or without TBBO 
was compared. Also, 1\% DMSO was used to dissolve test compounds and hence, the survival (\%) was represented as the control $=100$.

On the other hand, it was previously reported that the pulp-biocompatibility of 4-META/MMATBBO resins was responsible for the dentin-adhesive activity of this resin ${ }^{2,17)}$. The risks of pulpal toxicity of resin components are connected with their ability to diffuse through the dentine and accumulate in the pulp ${ }^{23)}$. The present studies are out of the scope of this problem but we have recently found the reduction of cytotoxic activity of 4-META/MMA in the presence of fetal bovine serum and human albumin (data not shown), possibly due to its interaction between 4-META/MMA and proteins.

\section{ACKNOWLEDGEMEN'TS}

This study was supported in part by a grant-in-aid for scientific research from the Ministry of Education, Science, Sports and Culture, Japan (No14571859).

\section{REFERENCES}

1) Masuhara E. A dental adhesive and its clinical applications. Tokyo: Quintessence; 1982, p 11-22.

2) Nakabayashi N, Kojima K, Masuhara E. The promotion of adhesion by the infiltration of monomers into tooth substrates. J Biomed Mater Res 1982; 16: 265273.

3) Kameyama A, Ihara S, Amagai T, Miyake $Y$, Kawada E, Oda Y, Yanagisawa T, Hirai Y. Adhesion of 4META/MMA-TBB resin to heated dentin: Effects of pretreatments with $\mathrm{FeCl}_{3}$ and/or HEMA. Dent Mater J2003, 22: 1-9.

4) Imai $Y$, Watanabe M, Lee, H-E, Kojima K, Kadoma Y. Cytotoxicity of monomers used dental resins. Report of the Institute for Medical and Lental Engineering 1988; 22: 87-90.

5) Yokoyama K, Imai K. Effect of dental luting cements from the view point of cell recovery. J J Dent Mater 1990; 9: 703-719.

6) Hikage S, Atsuta M, Sato A. Biological evaluation of biomaterials using cultured chicken embryo femurs (part 3). J J Dent Mater 1989; 8: 642-647.

7) Hikage S, Sato A, Suzuki S, Cox CF, Sakaguchi K. Cytotoxicity of dental resin monomers in the presence of S9 mix enzymes. Dental Material J 1999; 18: 76-86.

8) Itoh K, Mizushima T, Nakabayashi N. Pulp response of adhesive dental resins. J J Dent Mater 1986; 5: 287294.

9) Nakamura $M$, Inoue $T$, Shimono $M$. Immunohistochemical study of dental pulp applied with 4-META/
MMA adhesive resin after pulpotomy. J Biomed Mater Res 2000; 51: 241-248.

10) Fujisawa S, Kadoma Y, Komoda Y. Hemolytic activity of a dental adhesive monomer (4- methacryloyloxyethoxycarbonylphthalic anhydride, 4-META) and its interaction with phospholipid liposomes as determined by NMR and DSC. Dent Mater J 1991; 9: 18-26.

11) Fujisawa $S$, Atsumi $T$, Satoh $K$, Sakagami H. Interaction between 2-etoxybenzoic acid (EBA) and eugenol, and related changes in cytotoxicity. J Dent Res 2003; 82: 43-47.

12) Mosmann T. Rapid colorimetric assay for cellular growth and survival: Application to proliferation and cytotoxicity assays. J Immunol Meth 1983; 65: 55-63.

13) Ozaki M, Suzuki M, Itoh K, Wakumoto S, Hisamitsu $H$. Laser-Raman spectroscopic study of the adhesive interface. Analysis between 4-META/MMA-TBB resin and bovine or human dentin. Dental Materials J 1992; 11: 70-76.

14) Fujisawa S, Masuhara E. Binding of methyl methacrylate to bovine serum albumin, J Dent Res 1980; 59: 2056-2061.

15) Fujisawa S. Binding of mono-and dimethacrylates to human serum albumin using fluorescent probes. $\mathrm{J} J$ Dent Mater 1986; 5: 566-570.

16) Fujisawa $\mathrm{S}$, Ito $\mathrm{S}$. $1 \mathrm{H}-\mathrm{NMR}$ Studies of the interaction of dental adhesive monomer, 4-META with calcium. Dental Material J 1999; 18: 54-62.

17) Masuhara E. Uber die Chemie Eines Neuen Haftfahigen Kunststoff-Fullungmaterials. Dtsch zahnarztl Z 1969; 24: 620-628.

18) Laurence WH, Bass GE, Purcell WP, Autian J. Use of mathematical models in the study of structure-toxicity relationships of dental compounds: I. Esters of acrylic and methacrylic acid. J Dent Res 1972; 51: 526-535.

19) Yoshii Y. Cytotoxicity of effects of acrylates and methacrylates: Relationships of monomer structures and cytotoxicity. Biomed Mater Res 1997; 37: 517-524.

20) Fujisawa S, Imai Y, Kojima K, Masuhara E. Studies on hemolytic activity of bisphenol A Diglycidyl Methacrylate (BIS-GMA). J Dent Res 1978; 57: 98-102.

21) Fujisawa S, Masuhara E. Determination of hydrophobic parameters ( $\log \mathrm{P}_{\mathrm{HPLC}}$ ) for methacrylates, initiators and miscellaneous compounds used in dentistry by high-performance liquid chromatography (HPLC). J J Dent Mater 1981; 22: 277-284.

22) Fujisawa S, Atsumi T, Kadoma, Y. Cytotoxicity of methyl methacrylate (MMA) and related compounds and their interaction with biomembranes. Oral Diseases 2000; 6: 215-221.

23) Wataha JC, Hanks CT, Strawn SE, Fat JC. Cytotoxicity of components of resins and other dental restorative materials. J Oral Rehabil 1994; 21: 453-462. 